\title{
Stochastic Permanence, Stationary Distribution and Extinction of a Single-Species Nonlinear Diffusion System with Random Perturbation
}

\author{
Li Zu, ${ }^{1,2}$ Daqing Jiang, ${ }^{2}$ and Donal O'Regan ${ }^{3}$ \\ ${ }^{1}$ School of Science, Changchun University, Changchun 130022, China \\ ${ }^{2}$ School of Mathematics and Statistics, Northeast Normal University, Changchun 130024, China \\ ${ }^{3}$ School of Mathematics, Statistics and Applied Mathematics, National University of Ireland, Galway, Ireland \\ Correspondence should be addressed to Daqing Jiang; daqingjiang2010@hotmail.com
}

Received 5 October 2013; Accepted 22 November 2013; Published 22 January 2014

Academic Editor: Weiming Wang

Copyright $\odot 2014 \mathrm{Li} \mathrm{Zu}$ et al. This is an open access article distributed under the Creative Commons Attribution License, which permits unrestricted use, distribution, and reproduction in any medium, provided the original work is properly cited.

\begin{abstract}
We analyze the influence of stochastic perturbations on a single-species logistic model with the population's nonlinear diffusion among $n$ patches. First, we show that this system has a unique positive solution. Then we obtain sufficient conditions for stochastic permanence and persistence in mean, stationary distribution, and extinction. Finally, we illustrate our conclusions through numerical simulation.
\end{abstract}

\section{Introduction}

Spatial factors which play a fundamental role in persistence and evolution of species can be modeled by a diffusion process. We have two typical equations to model the diffusion process. One is semilinear parabolic equations, that is, reaction-diffusion systems, where the populations are continuously spread out in space. The other is discrete diffusion systems, where several species are distributed over an interconnected network of multiple patches and there are population migrations among patches. Allen [1] studied and investigated the logistic nonlinear directed diffusion model

$$
\dot{x}_{i}=x_{i}\left(a_{i}-b_{i} x_{i}\right)+\sum_{j=1, j \neq i}^{n} d_{i j}\left(x_{j}^{2}-\alpha_{i j} x_{i}^{2}\right), \quad i=1,2, \ldots, n,
$$

where $x_{i}$ denotes the density dependent growth rate in patch $i$. The constants $d_{i j}(i, j=1,2, \ldots, n, j \neq i)$ are the dispersal rate from the $j$ th patch to the $i$ th patch, and the nonnegative constant $\alpha_{i j}$ can be selected to represent different boundary conditions [2]. Allen proved that the system (1) has a unique positive solution on a maximal interval (see [3]) and is strongly persistent and the population size can increase without bound or bounded under reversed conditions (see [1]). The fundamental tools to prove these results are the cooperative system theory and the cooperative matrix [1-4]. For system (1), Lu and Takeuchi [2, Theorem 3] extended Allen's results and obtained the following necessary and sufficient conditions.

(i) The system (1) possesses a globally stable positive equilibrium point $\left(x_{1}^{*}, x_{2}^{*}, \ldots, x_{n}^{*}\right)$, if the largest eigenvalue of the cooperative negative matrix $A$ is less than 0 .

(ii) Every solution of the system is unbounded, if the above condition is not satisfied. 
Here

$$
A=\left(\begin{array}{cccc}
b_{1}+\sum_{j=1, j \neq 1}^{n} d_{1 j} \alpha_{1 j} & -d_{12} & \cdots & -d_{1 n} \\
-d_{21} & b_{2}+\sum_{j=1, j \neq 2}^{n} d_{2 j} \alpha_{2 j} & \cdots & -d_{2 n} \\
\vdots & \vdots & \ddots & \cdots \\
-d_{n 1} & -d_{n 2} & \cdots & b_{n}+\sum_{j=1, j \neq n}^{n} d_{n j} \alpha_{n j}
\end{array}\right) .
$$

Deterministic models are often subject to stochastic perturbations, and it is useful to reveal how the noise affects the population system. There are many papers which study differential equations with stochastic perturbations (see [510] and the references therein). Li et al. [7] studied the stochastic logistic populations system under regime switching and analyzed the asymptotic properties of their model. Jiang et al. $[8,9]$ investigated a logistic equation with random perturbation and obtained many results such as global stability and stochastic permanence. More investigations and improvements of these stochastic models can be found in [11, 12]. There is very little known on the dynamic behavior in the single-species dispersal system with stochastic perturbation.

Now we introduce randomly perturbation into the intrinsic growth rate $a_{i}$ and assume that parameters $a_{i}$ are disturbed to

$$
a_{i}+\sigma_{i} \dot{B}_{i}(t), \quad i=1,2, \ldots, n,
$$

where $B_{i}(t)$ is mutually independent Brownian motion and $\sigma_{i}$ is a positive constant representing the intensity of the white noise. Then the stochastic system takes the form

$$
\begin{array}{r}
d x_{i}=\left[x_{i}\left(a_{i}-b_{i} x_{i}\right)+\sum_{j=1, j \neq i}^{n} d_{i j}\left(x_{j}^{2}-\alpha_{i j} x_{i}^{2}\right)\right] d t+\sigma_{i} x_{i} d B_{i}(t), \\
i=1,2, \ldots, n .
\end{array}
$$

For convenience, let $\bar{b}_{i}=b_{i}+\sum_{j=1, j \neq i}^{n} d_{i j} \alpha_{i j}$ and $d_{i i}=0$. Thus, the equation is rewritten as

$$
\begin{array}{r}
d x_{i}=\left[x_{i}\left(a_{i}-\bar{b}_{i} x_{i}\right)+\sum_{j=1}^{n} d_{i j} x_{j}^{2}\right] d t+\sigma_{i} x_{i} d B_{i}(t), \\
i=1,2, \ldots, n .
\end{array}
$$

In this paper, we assume that $d_{i j}$ and $\alpha_{i j}$ are nonnegative constants, the parameters $a_{i}, b_{i}$ are positive constants, and so $\bar{b}_{i}>0$.

The rest of the paper is arranged as follows. We will show that there exists a unique positive global solution with any initial positive value in Section 2. In Section 3, we will investigate sufficient conditions for stochastic permanence and persistence in mean which are important in an ecological system. In a deterministic system, the global attractivity of the positive equilibrium is studied, but it is impossible to expect system (5) to tend to a steady state. We investigate the stationary distribution of this system by the Lyapunov functional technique. This can be considered as weak stability, which appears as the solution is fluctuating in a neighborhood of the point. In Section 4, we show that if the white noise is small, there is a stationary distribution of (5) and it has an ergodic property. Results on dynamic in a patchy environment have largely been restricted to extinction analysis which means that the population system will survive or die out in the future. In Section 5, we give sufficient conditions for extinction. In Sections 6 and 7, we make numerical simulation to confirm the effect of white noise intensity and the diffusion coefficient on the species and give a conclusion. Finally, for the completeness of the paper, we give an Appendix containing some results which will be used in other sections.

The key method used in this paper is the analysis of Lyapunov functions $[5,8-10,12]$.

Throughout this paper, unless otherwise specified, let $\left(\Omega,\left\{\mathscr{F}_{t}\right\}_{t>0}, P\right)$ be a complete probability space with a filtration $\left\{\mathscr{F}_{t}\right\}_{t \geq 0}$ satisfying the usual conditions (i.e., it is right continuous and $\mathscr{F}_{0}$ contains all $P$-null sets). Let $R_{+}^{n}$ is the positive cone of $R^{n}$, namely, $R_{+}^{n}=\left\{\left(x_{1}, x_{2}, \ldots, x_{n}\right) \in R^{n}\right.$ : $\left.x_{i}>0, i=1,2, \ldots, n\right\}$. For convenience and simplicity in the following discussion, denote $x(t)=\left(x_{1}(t), x_{2}(t), \ldots, x_{n}(t)\right)$ and $x_{i}=x_{i}(t)$. If $A$ is a vector or matrix, its transpose is denoted by $A^{T}$. By $A \gg 0$ we mean all elements of $A$ are positive. If $A$ is a matrix, its trace norm is denoted by $|A|=$ $\sqrt{\operatorname{trace}\left(A^{T} A\right)}$ whilst its operator norm is denoted by $\|A\|=$ $\sup \{|A x|:|x|=1\}$. We impose the following assumptions.

Assumption 1. $\bar{b}_{i}>\sum_{j=1}^{n} d_{j i}, i=1,2, \ldots, n$.

Assumption 2. $a_{i}>\sigma_{i}^{2} / 2, i=1,2, \ldots, n$.

\section{Positive and Global Solutions}

As the solution of $\operatorname{SDE}$ (5) has biological significance, it should be nonnegative. Moreover, in order for a stochastic differential equation to have a unique global (i.e., no explosion in a finite time) solution for any given initial 
value, the coefficients of the equation are generally required to satisfy a linear growth condition and a local Lipschitz condition (cf. Mao [13]). However, the coefficients of SDE (5) do not satisfy a linear growth condition, though they are locally Lipschitz continuous. In this section, we will use a method similar to Mao et al. [5, Theorem 2.1] to prove that the solution of SDE (5) is nonnegative and global.

Theorem 3. Let Assumption 1 hold. For any given initial value $x(0) \in R_{+}^{n}$, there is a unique positive solution $x(t)$ of system (5), and the solution will remain in $R_{+}^{n}$ with probability 1 .

Proof. Define a $C^{2}$-function $V: R_{+}^{n} \rightarrow R_{+}$by

$$
V(x)=\sum_{i=1}^{n}\left(x_{i}-1-\log x_{i}\right)
$$

The nonnegativity of this function can be observed from $a-$ $1-\log a \geq 0$ on $a>0$ with equality holding if and only if $a=1$. For $x \in R_{+}^{n}$, applying Itô's formula, we have

$d V(x)$

$$
\begin{aligned}
= & \sum_{i=1}^{n}\left[d x_{i}-\frac{1}{x_{i}} d x_{i}+\frac{1}{2 x_{i}^{2}}\left(d x_{i}\right)^{2}\right] \\
= & \sum_{i=1}^{n}\left[-\bar{b}_{i} x_{i}^{2}+\sum_{j=1}^{n} d_{i j} x_{j}^{2}+\left(a_{i}+\bar{b}_{i}\right) x_{i}-\sum_{j=1}^{n} d_{i j} \frac{x_{j}^{2}}{x_{i}}-a_{i}+\frac{\sigma_{i}^{2}}{2}\right] d t \\
& +\sum_{i=1}^{n} \sigma_{i}\left(x_{i}-1\right) d B_{i}(t) \\
\leq & \sum_{i=1}^{n}\left[\left(-\bar{b}_{i}+\sum_{j=1}^{n} d_{j i}\right) x_{i}^{2}+\left(a_{i}+\bar{b}_{i}\right) x_{i}-a_{i}+\frac{\sigma_{i}^{2}}{2}\right] d t \\
& +\sum_{i=1}^{n} \sigma_{i}\left(x_{i}-1\right) d B_{i}(t) \\
= & : L V d t+\sum_{i=1}^{n} \sigma_{i}\left(x_{i}-1\right) d B_{i}(t),
\end{aligned}
$$

where

$$
L V=\sum_{i=1}^{n}\left[\left(-\bar{b}_{i}+\sum_{j=1}^{n} d_{j i}\right) x_{i}^{2}+\left(a_{i}+\bar{b}_{i}\right) x_{i}-a_{i}+\frac{\sigma_{i}^{2}}{2}\right]
$$

and by Assumption 1, we know that there exists a positive constant number $K$ satisfying

$$
L V \leq K
$$

and $K$ is independent of $x_{i}$ and $t$. By a proof similar to Mao et al. [5, Theorem 2.1], we obtain the desired assertion.

\section{Stochastic Permanence and Persistence in Mean}

In this section, we will investigate the persistence under two different meanings: stochastic permanence and persistence in mean.

3.1. Stochastic Permanence. Theorem 3 shows that the solution of SDE (5) will remain in the positive cone $R_{+}^{n}$ with probability 1 . We now further discuss how the solution varies in $R_{+}^{n}$ in detail. We will first give the definitions of stochastically ultimate boundedness and stochastic permanence.

Definition 4. The SDE (5) is said to be stochastically ultimately bounded, if for any $\epsilon \in(0,1)$, there exist positive constants $\chi_{i}\left(=\chi_{i}(\epsilon)\right)(i=1,2, \ldots, n)$ such that for any initial value $x(0) \in R_{+}^{n}$, the solution of $\operatorname{SDE}$ (5) has the property that

$$
\limsup _{t \rightarrow \infty} P\left\{x_{i}(t)>\chi_{i}\right\}<\epsilon, \quad i=1,2, \ldots, n,
$$

where $\left(x_{1}(t), x_{2}(t), \ldots, x_{n}(t)\right)$ is the solution of SDE (5) with any initial value $x(0) \in R_{+}^{n}$.

Definition 5. The SDE (5) is said to be stochastically permanent, if for any $\epsilon \in(0,1)$, there are positive constants $\chi_{i}\left(=\chi_{i}(\epsilon)\right)$ and $\delta_{i}\left(=\delta_{i}(\epsilon)\right)(i=1,2, \ldots, n)$ such that

$$
\begin{array}{r}
\liminf _{t \rightarrow \infty} P\left\{x_{i}(t) \leq x_{i}\right\} \geq 1-\epsilon, \\
\liminf _{t \rightarrow \infty} P\left\{x_{i}(t) \geq \delta_{i}\right\} \geq 1-\epsilon, \\
i=1,2, \ldots, n .
\end{array}
$$

It is clear that if the system is stochastically permanent, it must be stochastically ultimately bounded.

Lemma 6. Under Assumption 1, for any given initial value $x(0) \in R_{+}^{n}$, there exists a positive constant $\kappa(p)$ such that the solution $x(t)$ of SDE (5) has the following property:

$$
E\left(\sum_{i=1}^{n} x_{i}(t)\right)^{p} \leq \kappa(p), \quad t \geq 0, p>1
$$

Proof. By Theorem 3, we know that the solution $x(t)$ with initial value $x(0) \in R_{+}^{n}$ will remain in $R_{+}^{n}$ with probability 1 . For any given positive constant $p>1$, define

$$
V(x(t))=\left(\sum_{i=1}^{n} x_{i}(t)\right)^{p}
$$


By Itô's formula, we find that

$$
\begin{aligned}
& d V=p\left(\sum_{i=1}^{n} x_{i}\right)^{p-1} d\left(\sum_{i=1}^{n} x_{i}\right) \\
& +\frac{p(p-1)}{2}\left(\sum_{i=1}^{n} x_{i}\right)^{p-2} d\left(\sum_{i=1}^{n} x_{i}\right)^{2} \\
& =\left[p\left(\sum_{i=1}^{n} x_{i}\right)^{p-1} \sum_{i=1}^{n}\left[x_{i}\left(a_{i}-\bar{b}_{i} x_{i}\right)+\sum_{j=1}^{n} d_{i j} x_{j}^{2}\right]\right. \\
& \left.+\frac{p(p-1)}{2}\left(\sum_{i=1}^{n} x_{i}\right)^{p-2} \sum_{i=1}^{n} \sigma_{i}^{2} x_{i}^{2}\right] d t \\
& +p\left(\sum_{i=1}^{n} x_{i}\right)^{p-1} \sum_{i=1}^{n} \sigma_{i} x_{i} d B_{i}(t) \\
& =p\left[\left(\sum_{i=1}^{n} x_{i}\right)^{p-1} \sum_{i=1}^{n}\left(-\bar{b}_{i}+\sum_{j=1}^{n} d_{j i}\right) x_{i}^{2}\right. \\
& +p\left(\sum_{i=1}^{n} x_{i}\right)^{p-1} \sum_{i=1}^{n}\left(a_{i} x_{i}\right) \\
& \left.+\frac{p(p-1)}{2}\left(\sum_{i=1}^{n} x_{i}\right)^{p-2} \sum_{i=1}^{n} \sigma_{i}^{2} x_{i}^{2}\right] d t \\
& +p\left(\sum_{i=1}^{n} x_{i}\right)^{p-1} \sum_{i=1}^{n} \sigma_{i} x_{i} d B_{i}(t) \\
& \leq\left[-\min _{1 \leq i \leq n}\left\{\frac{p\left(\bar{b}_{i}-\sum_{j=1}^{n} d_{j i}\right)}{n}\right\}\left(\sum_{i=1}^{n} x_{i}\right)^{p+1}\right. \\
& \left.+\max _{1 \leq i \leq n}\left\{p a_{i}+\frac{p(p-1)}{2} \sigma_{i}^{2}\right\}\left(\sum_{i=1}^{n} x_{i}\right)^{p}\right] d t \\
& +p\left(\sum_{i=1}^{n} x_{i}\right)^{p-1} \sum_{i=1}^{n} \sigma_{i} x_{i} d B_{i}(t) \\
& =:\left[-\widehat{\beta}\left(\sum_{i=1}^{n} x_{i}\right)^{p+1}+\check{\alpha}\left(\sum_{i=1}^{n} x_{i}\right)^{p}\right] d t \\
& +p\left(\sum_{i=1}^{n} x_{i}\right)^{p-1} \sum_{i=1}^{n} \sigma_{i} x_{i} d B_{i}(t),
\end{aligned}
$$

where $\widehat{\beta}=\min _{1 \leq i \leq n}\left\{p\left(\bar{b}_{i}-\sum_{j=1}^{n} d_{j i}\right) / n\right\}$, and $\check{\alpha}=\max _{1 \leq i \leq n}\left\{p a_{i}+\right.$ $\left.(p(p-1) / 2) \sigma_{i}^{2}\right\}$. It's clear that $\check{\alpha}>0$ and $\widehat{\beta}>0$. Hence we get

$$
\begin{aligned}
& \frac{d E\left(\sum_{i=1}^{n} x_{i}(t)\right)^{p}}{d t} \\
& \leq \check{\alpha} E\left(\sum_{i=1}^{n} x_{i}(t)\right)^{p}-\widehat{\beta}\left[E\left(\sum_{i=1}^{n} x_{i}(t)\right)^{p}\right]^{(p+1) / p} \\
& =E\left(\sum_{i=1}^{n} x_{i}(t)\right)^{p}\left\{\check{\alpha}-\widehat{\beta}\left[E\left(\sum_{i=1}^{n} x_{i}(t)\right)^{p}\right]^{1 / p}\right\} .
\end{aligned}
$$

Therefore, letting $z(t)=E\left(\sum_{i=1}^{n} x_{i}(t)\right)^{p}$, we have

$$
\frac{d z(t)}{d t} \leq z(t)\left[\check{\alpha}-\widehat{\beta} z^{1 / p}(t)\right] \text {. }
$$

Notice that the solution of equation

$$
\frac{d \bar{z}(t)}{d t}=\bar{z}(t)\left[\check{\alpha}-\widehat{\beta} \bar{z}^{1 / p}(t)\right]
$$

obeys

$$
\bar{z}(t) \longrightarrow\left(\frac{\check{\alpha}}{\widehat{\beta}}\right)^{p}, \quad \text { as } t \longrightarrow \infty
$$

Thus by the comparison argument we get

$$
\limsup _{t \rightarrow \infty} z(t) \leq\left(\frac{\check{\alpha}}{\widehat{\widehat{\beta}}}\right)^{p} .
$$

Then we have

$$
\limsup _{t \rightarrow \infty} E\left(\sum_{i=1}^{n} x_{i}(t)\right)^{p} \leq\left(\frac{\check{\alpha}}{\widehat{\beta}}\right)^{p}=: L(p),
$$

which implies that there exists a $T>0$, such that

$$
E\left(\sum_{i=1}^{n} x_{i}(t)\right)^{p} \leq 2 L(p), \quad t>T .
$$

In addition, $E\left(\sum_{i=1}^{n} x_{i}(t)\right)^{p}$ is continuous, so we have

$$
E\left(\sum_{i=1}^{n} x_{i}(t)\right)^{p} \leq C(p), \quad t \in[0, T] .
$$

Let $\kappa(p)=\max \{2 L(p), C(p)\}$, and therefore

$$
E\left(\sum_{i=1}^{n} x_{i}(t)\right)^{p} \leq \kappa(p), \quad t \geq 0, p>1 .
$$

This completes the proof.

Theorem 7. Under Assumption 1, solutions of SDE (5) are stochastically ultimately bounded.

The proof of Theorem 7 is a simple application of the Chebyshev inequality and Lemma 6.

Since the solution of SDE (5) is positive, by the classical comparison theorem of stochastic differential equations [14], we can obtain the lemma. 
Lemma 8. Let Assumptions 1 and 2 hold, and $x(t) \in R_{+}^{n}$ is the solution of SDE (5) with initial value $x(0) \in R_{+}^{n}$. Then $x(t)$ has the property that

$$
x_{i}(t) \geq \phi_{i}(t),
$$

where $\phi_{i}(t)(i=1,2, \ldots, n)$ are the solutions of the following equations:

$$
\begin{array}{r}
d \phi_{i}(t)=\phi_{i}(t)\left[\left(a_{i}-\bar{b}_{i} \phi_{i}(t)\right) d t+\sigma_{i} d B_{i}(t)\right], \\
\phi_{i}(0)=x_{i}(0), \\
i=1,2, \ldots, n .
\end{array}
$$

In view of $\mathrm{Li}$ et al. [7, Lemma 3.6], one sees that, if Assumption 2 holds, there exist positive constants $H_{i}$ and $\theta$ such that $a_{i}-((\theta+1) / 2) \sigma_{i}^{2}>0(i=1,2, \ldots, n)$ satisfying the following inequalities:

$$
\begin{gathered}
\limsup _{t \rightarrow \infty} E\left[\frac{1}{\left(\phi_{i}(t)\right)^{\theta}}\right] \leq H_{i}, \quad i=1,2, \ldots, n, \\
\liminf _{t \rightarrow \infty} \frac{\log \phi_{i}(t)}{\log t} \geq-\frac{1}{\theta} \quad \text { a.s. } i=1,2, \ldots, n .
\end{gathered}
$$

These, together with Lemma 8, then we have.

Lemma 9. Under Assumptions 1 and 2, the solution $x(t)$ of $S D E(5)$ with any initial value $x(0) \in R_{+}^{n}$ has the property that

$$
\begin{gathered}
\limsup _{t \rightarrow \infty} E\left[\frac{1}{\left(x_{i}(t)\right)^{\theta}}\right] \leq H_{i}, \quad i=1,2, \ldots, n, \\
\liminf _{t \rightarrow \infty} \frac{\log x_{i}(t)}{\log t} \geq-\frac{1}{\theta} \quad \text { a.s. } i=1,2, \ldots, n,
\end{gathered}
$$

where $H_{i}$ are positive constants and $\theta>0$ such that $a_{i}-((\theta+$ 1) $/ 2) \sigma_{i}^{2}>0, i=1,2, \ldots, n$.

Theorem 10. Under Assumptions 1 and 2, SDE (5) is stochastically permanent.

Proof. Let $x(t)$ be the solution of SDE (5) with any given positive initial value $x(0) \in R_{+}^{n}$. By Lemma 9, we have

$$
\limsup _{t \rightarrow \infty} E\left[\frac{1}{\left(x_{i}(t)\right)^{\theta}}\right] \leq H_{i}, \quad i=1,2, \ldots, n .
$$

For $x(t) \in R_{+}^{n}$ and for any $\epsilon>0$, let $\delta_{i}=\left(\epsilon / H_{i}\right)^{1 / \theta}$, we get the following:

$$
\begin{aligned}
P\left\{x_{i}(t)<\delta_{i}\right\} & =P\left\{\frac{1}{\left(x_{i}(t)\right)^{\theta}}>\frac{1}{\delta_{i}^{\theta}}\right\} \\
& \leq \frac{E\left[1 /\left(x_{i}(t)\right)^{\theta}\right]}{1 / \delta_{i}^{\theta}} \\
& \leq \delta_{i}^{\theta} H_{i}=\epsilon, \quad i=1,2, \ldots, n
\end{aligned}
$$

Hence

$$
\limsup _{t \rightarrow \infty} P\left\{x_{i}(t)<\delta_{i}\right\} \leq \epsilon, \quad i=1,2, \ldots, n,
$$

and this implies

$$
\liminf _{t \rightarrow \infty} P\left\{x_{i}(t) \geq \delta_{i}\right\} \geq 1-\epsilon, \quad i=1,2, \ldots, n .
$$

The other part of Definition 5 follows from Theorem 7.

3.2. Persistence in Mean. In this section, we will investigate persistence in mean. First we introduce one definition.

Definition 11. SDE (5) is said to be persistent in mean, if there exist positive constants $m_{i}, M_{i}(i=1,2, \ldots, n)$ such that the solution $x(t)$ of SDE (5) has the following property:

$$
\begin{aligned}
& \limsup _{t \rightarrow \infty} \frac{1}{t} \int_{0}^{t} x_{i}(s) d s \leq M_{i} \quad \text { a.s. } i=1,2, \ldots, n, \\
& \liminf _{t \rightarrow \infty} \frac{1}{t} \int_{0}^{t} x_{i}(s) d s \geq m_{i} \quad \text { a.s. } i=1,2, \ldots, n .
\end{aligned}
$$

From the result in [12], we know that

$$
\begin{array}{r}
\liminf _{t \rightarrow \infty} \frac{1}{t} \int_{0}^{t} \phi_{i}(s) d s=\frac{a_{i}-\sigma_{i}^{2} / 2}{\bar{b}_{i}}, \quad \lim _{t \rightarrow \infty} \frac{\log \phi_{i}(t)}{t}=0 \\
\text { a.s. } i=1,2, \ldots, n .
\end{array}
$$

Using the above conclusions, we get the following lemmas.

Lemma 12. Suppose that Assumptions 1 and 2 are satisfied. Then the solution $x(t)$ of SDE (5) with any initial value $x(0) \epsilon$ $R_{+}^{n}$ has the following property:

$$
\begin{array}{r}
\liminf _{t \rightarrow \infty} \frac{1}{t} \int_{0}^{t} x_{i}(s) d s \geq \frac{a_{i}-\sigma_{i}^{2} / 2}{\bar{b}_{i}}, \quad \liminf _{t \rightarrow \infty} \frac{\log x_{i}(t)}{t} \geq 0 \\
\text { a.s. } i=1,2, \ldots, n .
\end{array}
$$

Lemma 13. Let Assumption 1 hold. For any given initial value $x(0) \in R_{+}^{n}$, the solution $x(t)$ of SDE (5) has the property that

$$
\limsup _{t \rightarrow \infty} \frac{\log \left[\sum_{i=1}^{n} x_{i}(t)\right]}{\log t} \leq 1 \quad \text { a.s. } i=1,2, \ldots, n
$$

Proof. Define $V: R_{+}^{n} \rightarrow R_{+}$by

$$
V(x(t))=\sum_{i=1}^{n} x_{i}(t)
$$


and applying the Itô's formula, one can see that

$$
\begin{aligned}
E\left[\sup _{t \leq u \leq t+1} V(x(u))\right] & \\
\leq & E[V(x(t))]+\check{a} \int_{t}^{t+1} E\left[\sum_{i=1}^{n} x_{i}(s)\right] d s \\
& +\check{b} \int_{t}^{t+1} E\left[\sum_{i=1}^{n} x_{i}^{2}(s)\right] d s \\
& +E\left[\sup _{t \leq u \leq t+1} \int_{t}^{u} \sum_{i=1}^{n} \sigma_{i} x_{i}(s) d B_{i}(s)\right] ;
\end{aligned}
$$

here $\check{a}=\max _{1 \leq i \leq n}\left\{a_{i}\right\}, \check{b}=\max _{1 \leq i \leq n}\left\{\left|-\bar{b}_{i}+\sum_{j=1}^{n} d_{j i}\right|\right\}$. From (12) of Lemma 6, we have

$$
\begin{gathered}
\limsup _{t \rightarrow \infty} E[V(x(t))]=\underset{t \rightarrow \infty}{\limsup E}\left[\sum_{i=1}^{n} x_{i}(t)\right] \leq[\kappa(2)]^{1 / 2}, \\
\limsup _{t \rightarrow \infty} \int_{t}^{t+1} E\left[\sum_{i=1}^{n} x_{i}^{2}(s)\right] d s \leq \kappa(2) .
\end{gathered}
$$

An application of the Burkholder-Davis-Gundy inequality (see $[12,14])$ and the Hölder inequality (see [12]) yields

$$
\begin{aligned}
& E\left[\sup _{t \leq u \leq t+1} \int_{t}^{u} \sum_{i=1}^{n} \sigma_{i} x_{i}(s) d B_{i}(s)\right] \\
& \quad \leq 3 \max _{1 \leq i \leq n}\left\{\sigma_{i}\right\} E\left(\int_{t}^{t+1}\left[\sum_{i=1}^{n} x_{i}^{2}(s)\right] d s\right)^{1 / 2} \\
& \leq 3 \check{\sigma}[\kappa(2)]^{1 / 2},
\end{aligned}
$$

where $\check{\sigma}=\max _{1 \leq i \leq n}\left\{\sigma_{i}\right\}$. This together with (39) yields

$$
\begin{aligned}
& \limsup _{t \rightarrow \infty} E\left[\sup _{t \leq u \leq t+1} V(x(u))\right] \\
& \quad \leq(1+\check{a}+3 \check{\sigma})[\kappa(2)]^{1 / 2}+\check{b} \kappa(2) .
\end{aligned}
$$

We observe from (41) that there is a positive constant $K^{*}$ such that

$$
E\left(\sup _{t \leq u \leq t+1}\left[\sum_{i=1}^{n} x_{i}(u)\right]\right) \leq K^{*} .
$$

Let $\epsilon>0$ be arbitrary. Then, by the well-known Chebyshev inequality, we have

$$
P\left\{\sup _{t \leq u \leq t+1}\left[\sum_{i=1}^{n} x_{i}(u)\right]>m^{1+\epsilon}\right\} \leq \frac{K^{*}}{m^{1+\epsilon}}, \quad m=1,2, \ldots
$$

Applying the Borel-Cantelli lemma (see [12]), for almost all $\omega \in \Omega$, we obtain that

$$
\sup _{t \leq u \leq t+1}\left[\sum_{i=1}^{n} x_{i}(u)\right] \leq m^{1+\epsilon}
$$

holds for all but finitely many $m$. Hence, we have a $m_{0}(\omega)$ such that (44) holds whenever $m \geq m_{0}$, for almost all $\omega \in \Omega$. Consequently, for almost all $\omega \in \Omega$, if $m \geq m_{0}$ and $m \leq t \leq$ $m+1$, we have

$$
\frac{\log \left[\sum_{i=1}^{n} x_{i}(t)\right]}{\log t} \leq \frac{(1+\epsilon) \log m}{\log m}=1+\epsilon
$$

Therefore

$$
\limsup _{t \rightarrow \infty} \frac{\log \left[\sum_{i=1}^{n} x_{i}(t)\right]}{\log t} \leq 1+\epsilon \quad \text { a.s. }
$$

Letting $\epsilon \rightarrow 0$ we obtain the desired assertion (36).

Theorem 14. Under Assumptions 1 and 2, for any given initial value $x(0) \in R_{+}^{n}$, the solution $x(t)$ of SDE (5) is persistent in mean.

Proof. Assume that $V: R_{+}^{n} \rightarrow R_{+}$is defined as in (37). From the inequality (28) of Lemma 9 and (36) of Lemma 13, one can derive that

$$
\lim _{t \rightarrow \infty} \frac{\log V(x(t))}{t}=0 \text { a.s. }
$$

By virtue of the Itô's formula and the Cauchy inequality, we have

$$
\begin{aligned}
& d \log V=\frac{1}{V} \sum_{i=1}^{n}\left[a_{i} x_{i}-\left(\bar{b}_{i}-\sum_{j=1}^{n} d_{j i}\right) x_{i}^{2}\right] d t \\
& -\frac{1}{2 V^{2}} \sum_{i=1}^{n} \sigma_{i}^{2} x_{i}^{2} d t+\frac{1}{V} \sum_{i=1}^{n} \sigma_{i} x_{i} d B_{i}(t) \\
& =\left[\frac{1}{V} \sum_{i=1}^{n} a_{i} x_{i}-\frac{1}{2 V^{2}} \sum_{i=1}^{n} \sigma_{i}^{2} x_{i}^{2}\right] d t \\
& -\frac{1}{V} \sum_{i=1}^{n}\left(\bar{b}_{i}-\sum_{j=1}^{n} d_{j i}\right) x_{i}^{2} d t+\frac{1}{V} \sum_{i=1}^{n} \sigma_{i} x_{i} d B_{i}(t) \\
& \leq\left[\max _{1 \leq i \leq n}\left\{a_{i}\right\}-\frac{\sum_{i=1}^{n} \sigma_{i}^{2} x_{i}^{2}}{2\left(\sum_{i=1}^{n}\left(1 / \sigma_{i}^{2}\right)\right)\left(\sum_{i=1}^{n} \sigma_{i}^{2} x_{i}^{2}\right)}\right] d t \\
& -\frac{1}{n} \min _{1 \leq i \leq n}\left\{\bar{b}_{i}-\sum_{j=1}^{n} d_{j i}\right\} \frac{\left(\sum_{i=1}^{n} x_{i}\right)^{2}}{\sum_{i=1}^{n} x_{i}} d t \\
& +\frac{1}{V} \sum_{i=1}^{n} \sigma_{i} x_{i} d B_{i}(t) \\
& =:\left(\check{a}-\frac{\widehat{\sigma}^{2}}{2}\right) d t-\frac{\widehat{b}}{n} \sum_{i=1}^{n} x_{i} d t+\frac{1}{V} \sum_{i=1}^{n} \sigma_{i} x_{i} d B_{i}(t) .
\end{aligned}
$$


Here $\check{a}=\max _{1 \leq i \leq n}\left\{a_{i}\right\}, \widehat{\sigma}^{2} / 2=1 /\left(2\left(\sum_{i=1}^{n}\left(1 / \sigma_{i}^{2}\right)\right)\right)$ and $\widehat{b}=$ $\min _{1 \leq i \leq n}\left\{\bar{b}_{i}-\sum_{j=1}^{n} d_{j i}\right\}$. Integrating both sides of the above inequality (48) from 0 to $t$ gives

$$
\begin{aligned}
\log V(x(t))+\frac{\widehat{b}}{n} \int_{0}^{t} \sum_{i=1}^{n} x_{i}(s) d s \\
\quad \leq \log V(x(0))+\int_{0}^{t}\left(\check{a}-\frac{\widehat{\sigma}^{2}}{2}\right) d s+M(t),
\end{aligned}
$$

where $M(t)$ is a martingale defined by

$$
M(t)=\int_{0}^{t} \frac{\sum_{i=1}^{n} \sigma_{i} x_{i}(s) d B_{i}(s)}{\sum_{i=1}^{n} x_{i}(s)}
$$

with $M(0)=0$. The quadratic variation of this martingale is

$$
\langle M, M\rangle_{t}=\int_{0}^{t} \frac{\sum_{i=1}^{n} \sigma_{i}^{2} x_{i}^{2}(s)}{\left(\sum_{i=1}^{n} x_{i}(s)\right)^{2}} d s \leq \max _{1 \leq i \leq n}\left\{\sigma_{i}^{2}\right\} t .
$$

By the strong law of large numbers for martingales (see [11]), we have

$$
\lim _{t \rightarrow \infty} \frac{M(t)}{t}=0 \quad \text { a.s. }
$$

It finally follows from (49) by dividing by $t$ on both sides and then letting $t \rightarrow \infty$; that is,

$$
\frac{\widehat{b}}{n} \limsup _{t \rightarrow \infty} \frac{1}{t} \int_{0}^{t} \sum_{i=1}^{n} x_{i}(s) d s \leq \check{a}-\frac{\widehat{\sigma}^{2}}{2} \quad \text { a.s. }
$$

which implies that

$$
\limsup _{t \rightarrow \infty} \frac{1}{t} \int_{0}^{t} \sum_{i=1}^{n} x_{i}(s) d s \leq \frac{n}{\widehat{b}}\left(\check{a}-\frac{\widehat{\sigma}^{2}}{2}\right) \quad \text { a.s. }
$$

On the other hand, from Lemma 12, we know that

$$
\liminf _{t \rightarrow \infty} \frac{1}{t} \int_{0}^{t} x_{i}(s) d s \geq \frac{1}{\bar{b}_{i}}\left(a_{i}-\frac{\sigma_{i}^{2}}{2}\right) \quad \text { a.s. } i=1,2, \ldots, n .
$$

Let $M_{i}=(n / \widehat{b})\left(\check{a}-\left(\widehat{\sigma}^{2} / 2\right)\right), m_{i}=\left(1 / \bar{b}_{i}\right)\left(a_{i}-\left(\sigma_{i}^{2} / 2\right)\right)(i=$ $1,2, \ldots, n)$, then we have

$$
\begin{array}{r}
\limsup _{t \rightarrow \infty} \frac{1}{t} \int_{0}^{t} x_{i}(s) d s \leq M_{i}, \quad \liminf _{t \rightarrow \infty} \frac{1}{t} \int_{0}^{t} x_{i}(s) d s \geq m_{i} \\
\text { a.s. } i=1,2, \ldots, n .
\end{array}
$$

Thus the required assertion follows.

\section{Stationary Distribution}

In this section, we investigate that there is a stationary distribution for SDE (5) instead of asymptotically stable equilibria. In order to ensure that system (1) has a globally stable positive equilibrium point $x^{*}=\left(x_{1}^{*}, x_{2}^{*}, \ldots, x_{n}^{*}\right)$, we need to introduce the following lemmas.
Lemma 15 (Mao and Yuan [11, Lemma 5.3]). If $B=\left(b_{i j}\right) \in$ $Z^{n \times n}$ has all of its row sums positive,

$$
\sum_{j=1}^{n} b_{i j}>0 \quad \forall 1 \leq i \leq n
$$

then $\operatorname{det} B>0$, where $Z^{n \times n}=\left\{B=\left(b_{i j}\right)_{n \times n}: b_{i j} \leq 0, i \neq j\right\}$.

Lemma 16 (Mao and Yuan [11, Theorem 2.10]). If $B \in Z^{n \times n}$, then the following statements are equivalent:

(a) $B$ is a nonsingular M-matrix;

(b) for any $y \gg 0$ in $R^{n}$, the linear equation $B x=y$ has $a$ unique solution $x \gg 0$;

(c) all of the principal minors of $B$ are positive; that is,

$$
L_{B}=\left|\begin{array}{cccc}
b_{11} & b_{12} & \ldots & b_{1 k} \\
b_{21} & b_{22} & \ldots & b_{2 k} \\
\vdots & \vdots & \ddots & \ldots \\
b_{k 1} & b_{k 2} & \ldots & b_{k k}
\end{array}\right|>0 \text { for every } k=1,2, \ldots, n
$$

(d) $B$ is positive stable; that is, the real part of each eigenvalue of $B$ is positive.

Let the matrix $A$ be defined as in Section 1 which can be simply written as

$$
A=\left(\begin{array}{cccc}
\bar{b}_{1} & -d_{12} & \ldots & -d_{1 n} \\
-d_{21} & \bar{b}_{2} & \ldots & -d_{2 n} \\
\vdots & \vdots & \ddots & \ldots \\
-d_{n 1} & d_{n 2} & \ldots & \bar{b}_{n}
\end{array}\right)
$$

Lemma 17. If Assumption 1 holds, then both $A$ and $A^{T}$ are nonsingular M-matrices.

Proof. We can obtain by Lemma 15 that if $\bar{b}_{i}>\sum_{j=1}^{n} d_{j i}(i=$ $1,2, \ldots, n)$, then all of the principal minors of $A^{T}$ are positive, and from (a) and (c) of Lemma 16, we know that $A^{T}$ is a nonsingular M-matrix.

Since all of the principal minors of $A$ and $A^{T}$ are the same, so $A$ is also a nonsingular $M$-matrix.

From Lemma 17, we know that if $A$ is a nonsingular $M$-matrix, then the real part of each eigenvalue of $A$ is positive based on (a) and (d) of Lemma 16, and we can also deduce that the maximum of the real part eigenvalues of negative matrix $A$ is less than 0 . This together with Theorem 3 of [2] stated in Section 1, we know that system (1) possesses a globally stable positive equilibrium point $x^{*}=$ $\left(x_{1}^{*}, x_{2}^{*}, \ldots, x_{n}^{*}\right)$ satisfying the equations

$$
a_{i} x_{i}^{*}-\bar{b}_{i} x_{i}^{* 2}+\sum_{j=1}^{n} d_{i j} x_{j}^{* 2}=0, \quad i=1,2, \ldots, n,
$$

where $x_{i}^{*}$ are positive constants. 
Theorem 18. Let Assumption 1 hold. Let $\delta_{2}=(1 / 2) \sum_{i=1}^{n} \sigma_{i}^{2} x_{i}^{*}$ and $\delta_{2}<\min _{1 \leq i \leq n}\left\{\left(\bar{b}_{i}-\sum_{i=1}^{n} d_{j i}\right) x_{i}^{* 2}\right\}$. Then there is a stationary distribution $\mu(\cdot)$ for $S D E(5)$ and it has the ergodic property.

Proof. Define $V: E_{l}=R_{+}^{n} \rightarrow R_{+}$by

$$
V(x)=\sum_{i=1}^{n}\left(x_{i}-x_{i}^{*}-x_{i}^{*} \log \frac{x_{i}}{x_{i}^{*}}\right) .
$$

By Itô's formula, we have

$$
L V=\sum_{i=1}^{n}\left[\left(x_{i}-x_{i}^{*}\right)\left(a_{i}-\bar{b}_{i} x_{i}+\sum_{j=1}^{n} d_{i j} \frac{x_{j}^{2}}{x_{i}}\right)+\frac{1}{2} \sigma_{i}^{2} x_{i}^{*}\right] .
$$

From (60), we know that

$$
a_{i}=\bar{b}_{i} x_{i}^{*}-\sum_{j=1}^{n} d_{i j} \frac{x_{j}^{* 2}}{x_{i}^{*}} .
$$

Substituting (63) into (62) one sees that

$$
\begin{aligned}
L V=\sum_{i=1}^{n}( & \left(x_{i}-x_{i}^{*}\right) \\
& \times\left[\bar{b}_{i}\left(x_{i}^{*}-x_{i}\right)+\sum_{j=1}^{n} d_{i j}\left(\frac{x_{j}^{2}}{x_{i}}-\frac{x_{j}^{* 2}}{x_{i}^{*}}\right)\right] \\
& \left.+\frac{1}{2} \sigma_{i}^{2} x_{i}^{*}\right) \\
=\sum_{i=1}^{n}\left[\begin{array}{l}
- \\
\end{array}\right. & \\
& +\sum_{j=1}^{n} d_{i j}\left(x_{i}-x_{i}^{*}\right)^{2} \\
& \left.+\frac{1}{2} \sigma_{i}^{2} x_{i}^{*}\right] .
\end{aligned}
$$

Using the inequality $a^{2}+b^{2} \geq 2 a b$, we compute $-\left(x_{i} x_{j}^{* 2} / x_{i}^{*}\right)-$ $\left(x_{i}^{*} x_{j}^{2} / x_{i}\right) \leq-2 x_{j} x_{j}^{*}$, and from the above inequality, we have

$$
\begin{aligned}
L V \leq \sum_{i=1}^{n}[ & -\bar{b}_{i}\left(x_{i}-x_{i}^{*}\right)^{2} \\
& \left.\quad+\sum_{j=1}^{n} d_{i j}\left(x_{j}^{2}-2 x_{j} x_{j}^{*}+x_{j}^{* 2}\right)+\frac{1}{2} \sigma_{i}^{2} x_{i}^{*}\right]
\end{aligned}
$$

$$
\begin{aligned}
& =\sum_{i=1}^{n}\left[-\bar{b}_{i}\left(x_{i}-x_{i}^{*}\right)^{2}+\sum_{j=1}^{n} d_{j i}\left(x_{i}-x_{i}^{*}\right)^{2}+\frac{1}{2} \sigma_{i}^{2} x_{i}^{*}\right] \\
& =-\sum_{i=1}^{n}\left(\bar{b}_{i}-\sum_{j=1}^{n} d_{j i}\right)\left(x_{i}-x_{i}^{*}\right)^{2}+\sum_{i=1}^{n} \frac{1}{2} \sigma_{i}^{2} x_{i}^{*} \\
& =:-\sum_{i=1}^{n}\left(\bar{b}_{i}-\sum_{j=1}^{n} d_{j i}\right)\left(x_{i}-x_{i}^{*}\right)^{2}+\delta_{2},
\end{aligned}
$$

where $\delta_{2}=\sum_{i=1}^{n}(1 / 2) \sigma_{i}^{2} x_{i}^{*}$. By Assumption 1, we know that the quadratic coefficients are less than zero. The following proof of ergodicity is similar to Theorem 3.2 in [10]. Note that $\delta_{2}<\min _{1 \leq i \leq n}\left\{\left(\bar{b}_{i}-\sum_{i=1}^{n} d_{j i}\right) x_{i}^{* 2}\right\}$; then the ellipse

$$
-\sum_{i=1}^{n}\left(\bar{b}_{i}-\sum_{j=1}^{n} d_{j i}\right)\left(x_{i}-x_{i}^{*}\right)^{2}+\delta_{2}=0
$$

lies entirely in $R_{+}^{n}$.

We can take $U$ to be a neighborhood of the ellipsoid with $\bar{U} \subset E_{l}=R_{+}^{n}$, so for $x \in E_{l} \backslash U, L V \leq-N$ ( $N$ is a positive constant), which implies that the condition (B2) in Assumption A.1 (see the Appendix) is satisfied. By Remark A.3 and Lemma A.4 and using the similar method as [10], we can prove that (A1) is also satisfied (see page 349 of [15]). Therefore, the stochastic system (5) has a stable stationary distribution $\mu(\cdot)$ and it is ergodic.

\section{Extinction}

We know that, if Assumption 1 holds, the solution of ODE (1) converges to a positive equilibrium point or is unbounded, so the population will not become extinct, and by Theorem 10, we note that if the condition $a_{i}>\sigma_{i}^{2} / 2(i=1,2, \ldots, n)$ is also satisfied, that is, the white noise intensity is smaller, then the species will be stochastically permanent and persistent in mean. We will show in this section that if the noise is sufficiently large, the solution to the associated SDE (5) will become extinct with probability 1 .

Theorem 19. Let Assumption 1 hold. Let $\check{a}=\max _{1 \leq i \leq n}\left\{a_{i}\right\}$ and $\widehat{\sigma}^{2} / 2=1 /\left(2\left(\sum_{i=1}^{n}\left(1 / \sigma_{i}^{2}\right)\right)\right)$. For any given initial value $x(0) \epsilon$ $R_{+}^{n}$, the solution of the SDE (5) has the property that

$$
\limsup _{t \rightarrow \infty} \frac{\log \left(\sum_{i=1}^{n} x_{i}(t)\right)}{t} \leq \check{a}-\frac{\widehat{\sigma}^{2}}{2} \quad \text { a.s. }
$$

Particularly, if $\check{a}-\left(\widehat{\sigma}^{2} / 2\right)<0$, then $\lim _{t \rightarrow \infty} x(t)=0$ a.s.

Proof. Define $V: R_{+}^{n} \rightarrow R_{+}$as in (37). Using Itô's formula, one can derive that

$$
d V=\sum_{i=1}^{n}\left[a_{i} x_{i}-\left(\bar{b}_{i}-\sum_{j=1}^{n} d_{j i}\right) x_{i}^{2}\right] d t+\sum_{i=1}^{n} \sigma_{i} x_{i} d B_{i}(t) .
$$



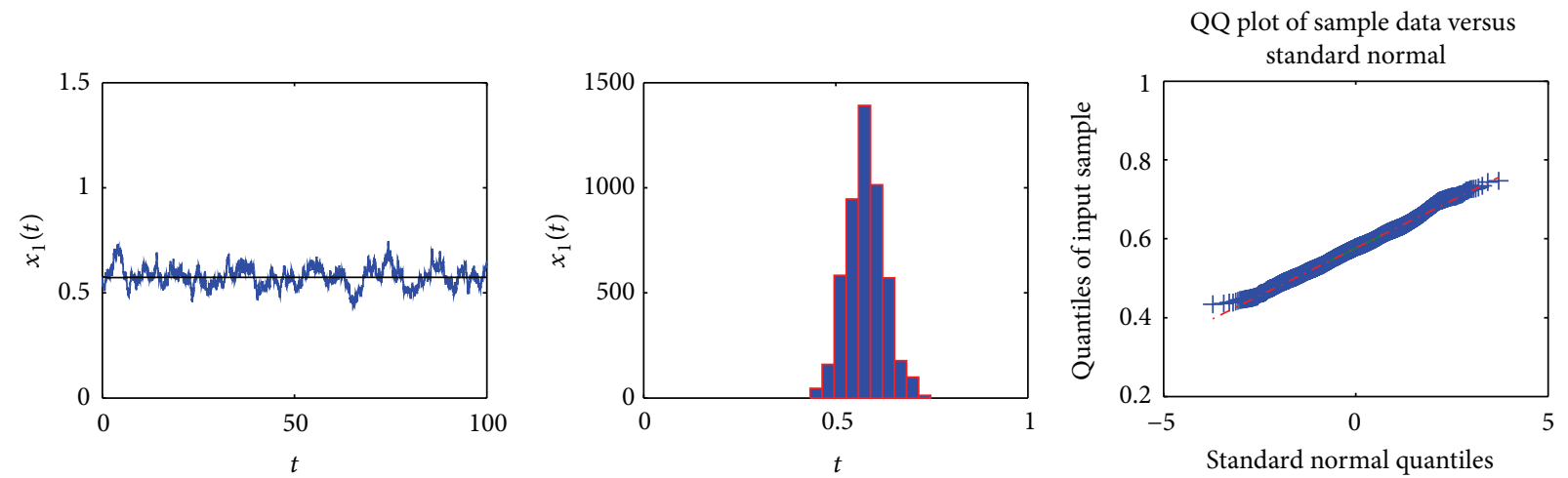

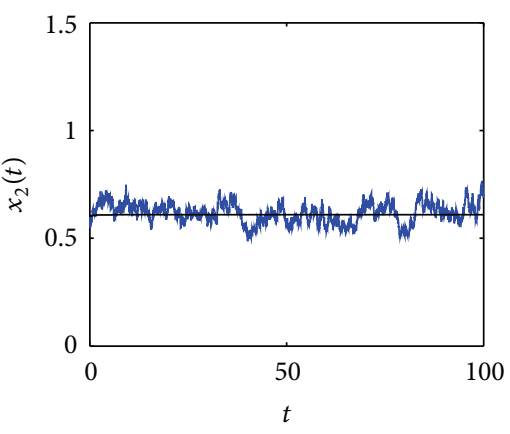

(a)

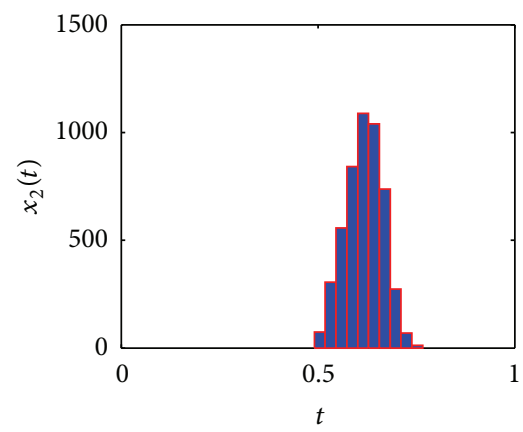

(b)

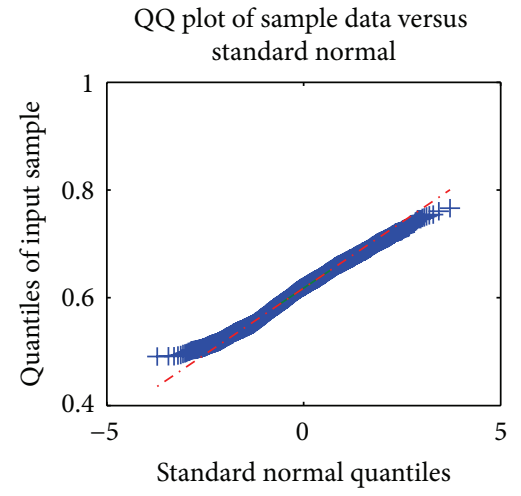

(c)

FIGURE 1: The pictures on the left are the solutions of stochastic system (73) and the corresponding undisturbed system, and the blue lines and the black lines represent them, respectively. The middle of the subgraphs is the histogram of stochastic system (73) and the subgraphs on the right are normal quantile-quantile plots of the values of the paths $x_{1}(t)$ and $x_{2}(t)$. The stochastic system is stochastically permanent and has a stationary distribution. $\sigma_{1}=0.1, \sigma_{2}=0.09$.

Following the scaling method of (48) and applying the Cauchy inequality and Assumption 1, we find

$$
\begin{aligned}
d \log V= & \frac{1}{V} \sum_{i=1}^{n}\left[a_{i} x_{i}-\left(\bar{b}_{i}-\sum_{j=1}^{n} d_{j i}\right) x_{i}^{2}\right] d t \\
& -\frac{1}{2 V^{2}} \sum_{i=1}^{n} \sigma_{i}^{2} x_{i}^{2} d t+\frac{1}{V} \sum_{i=1}^{n} \sigma_{i} x_{i} d B_{i}(t) \\
\leq & {\left[\frac{1}{V} \sum_{i=1}^{n} a_{i} x_{i}-\frac{1}{2 V^{2}} \sum_{i=1}^{n} \sigma_{i}^{2} x_{i}^{2}\right] d t } \\
& +\frac{1}{V} \sum_{i=1}^{n} \sigma_{i} x_{i} d B_{i}(t) \\
= & \left(\check{a}-\frac{\widehat{\sigma}^{2}}{2}\right) d t+\frac{1}{V} \sum_{i=1}^{n} \sigma_{i} x_{i} d B_{i}(t) .
\end{aligned}
$$

Integrating both sides of the above inequality (69) from 0 to $t$ gives

$$
\log V(x(t)) \leq \log V(x(0))+\int_{0}^{t}\left(\check{a}-\frac{\widehat{\sigma}^{2}}{2}\right) d s+M(t),
$$

where $M(t)$ is a martingale defined in the proof of Theorem 14. By the strong law of large numbers for martingales (see [11]), we have

$$
\lim _{t \rightarrow \infty} \frac{M(t)}{t}=0 \quad \text { a.s. }
$$

It finally follows from (70) by dividing by $t$ on both sides and then letting $t \rightarrow \infty$; that is,

$$
\limsup _{t \rightarrow \infty} \frac{\log V}{t} \leq \limsup _{t \rightarrow \infty} \frac{1}{t} \int_{0}^{t}\left(\check{a}-\frac{\widehat{\sigma}^{2}}{2}\right) d s=\check{a}-\frac{\widehat{\sigma}^{2}}{2} \quad \text { a.s. }
$$

Thus the required assertion follows.

\section{Numerical Simulation}

For the purpose of discussing the results, we consider the single-species nonlinear dispersal system with $n=2$. Assume that $\alpha_{i j}=1(i, j=1,2)$ and then $\bar{b}_{1}=b_{1}+d_{12}, \bar{b}_{2}=b_{2}+d_{21}$, so the SDE (5) becomes

$$
\begin{aligned}
& d x_{1}=\left[x_{1}\left(a_{1}-\bar{b}_{1} x_{1}\right)+d_{12} x_{2}^{2}\right] d t+\sigma_{1} x_{1} d B_{1}(t), \\
& d x_{2}=\left[x_{2}\left(a_{2}-\bar{b}_{2} x_{2}\right)+d_{21} x_{1}^{2}\right] d t+\sigma_{2} x_{2} d B_{2}(t) .
\end{aligned}
$$



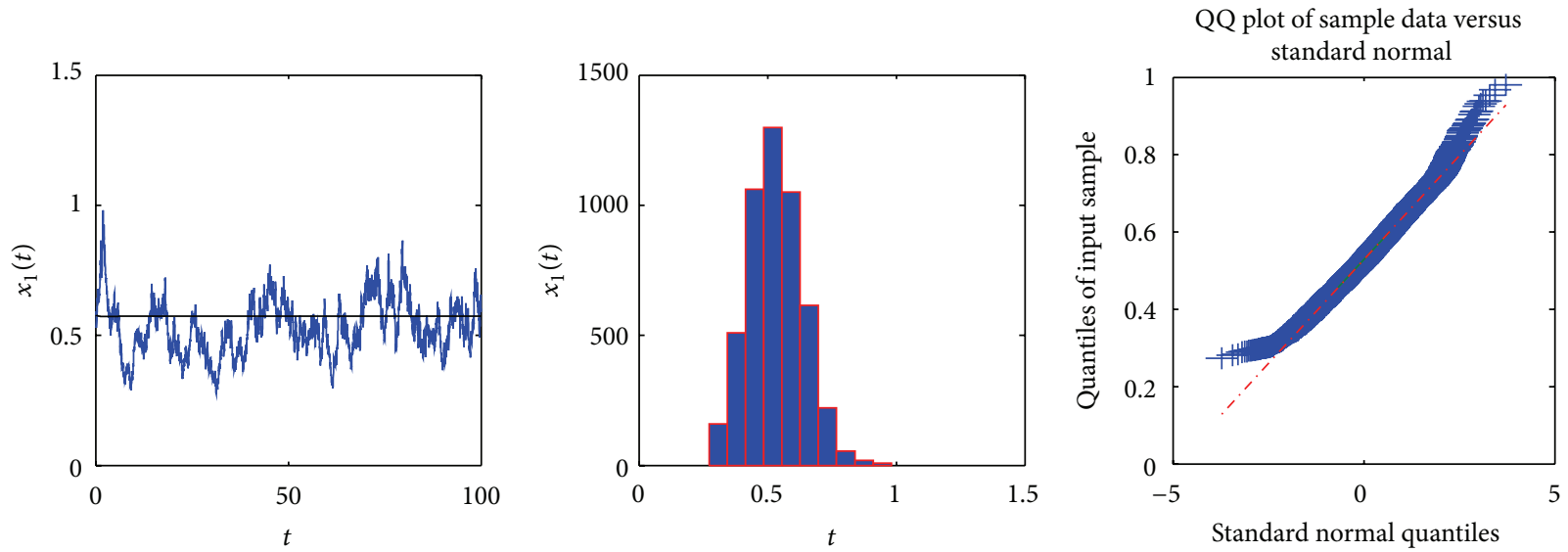

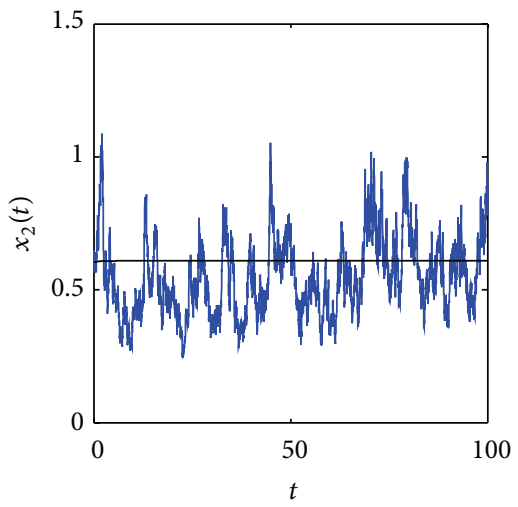

(a)

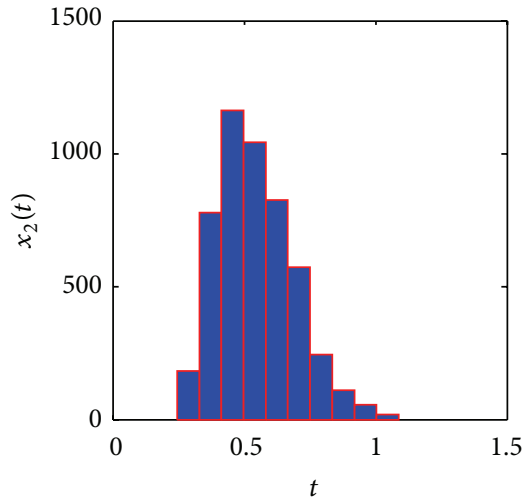

(b)

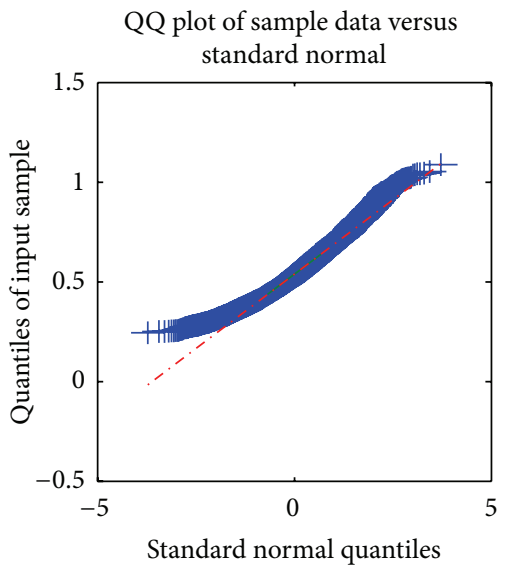

(c)

FIgURE 2: The subgraphs are defined as in Figure 1. $\sigma_{1}=0.2, \sigma_{2}=0.3$. The stochastic system is stochastically permanent and persistent in mean and has a stationary distribution.

We numerically simulate the solution of (73). By the method mentioned in [16], we consider the discretized equation

$$
\begin{aligned}
x_{1, k+1}= & x_{1, k}+\left[x_{1, k}\left(a_{1}-\bar{b}_{1} x_{1, k}\right)+d_{12} x_{2, k}^{2}\right] h \\
& +\sigma_{1} x_{1, k} \sqrt{h} \xi_{1, k}+\frac{1}{2} \sigma_{1}^{2} x_{1, k}\left(h \xi_{1, k}^{2}-h\right), \\
x_{2, k+1}= & x_{2, k}+\left[x_{2, k}\left(a_{2}-\bar{b}_{2} x_{2, k}\right)+d_{21} x_{1, k}^{2}\right] h \\
& +\sigma_{2} x_{2, k} \sqrt{h} \xi_{2, k}+\frac{1}{2} \sigma_{2}^{2} x_{2, k}\left(h \xi_{2, k}^{2}-h\right) .
\end{aligned}
$$

We will use the numerical simulation method and the help of Matlab software to illustrate our results. Choose $a_{1}=$ $0.3, a_{2}=0.4, b_{1}=b_{2}=0.6$. Assume that $d_{12}=0.6, d_{21}=0.5, \bar{b}_{1}=$ $1.2, \bar{b}_{2}=1.1$ in Figures 1, 2, 3, 4, 6(a), and 7 except in Figures 5 and 6(b) (in Figures 5 and 6(b), we choose $d_{12}=0.01, d_{21}=0$ for the purpose of illustrating the impact of different diffusion coefficients on population), the initial value $\left(x_{1}(0), x_{2}(0)\right)=$ $(0.58,0.60)$, and time step $h=0.01$. Then Assumption 1 is satisfied, so the corresponding deterministic model has a globally stable positive equilibrium point $x^{*}=\left(x_{1}^{*}, x_{2}^{*}\right) \doteq$ $(0.5734,0.6090)$. Obviously, by Theorem 3 , system (73) has a unique positive solution. The following discussion will be divided into two cases.

Case 1. The effect of different white noise intensity on the population.

In Figure 1, we choose $\sigma_{1}=0.1, \sigma_{2}=0.09$. Obviously Assumption 2 holds and the $\operatorname{SDE}(73)$ is stochastically permanent and persistent in mean. We compute $\delta_{2}=(1 / 2) \sigma_{1}^{2} x_{1}^{*}+$ $(1 / 2) \sigma_{2}^{2} x_{2}^{*} \doteq 5.3334 * 10^{-3}$, and $\min \left\{\left(\bar{b}_{1}-d_{21}\right)\left(x_{1}^{*}\right)^{2},\left(\bar{b}_{2}-\right.\right.$ $\left.\left.d_{12}\right)\left(x_{2}^{*}\right)^{2}\right\} \doteq 0.18544$, so the condition $\delta_{2}<\min \left\{\left(\bar{b}_{1}-\right.\right.$ $\left.\left.d_{21}\right)\left(x_{1}^{*}\right)^{2},\left(\bar{b}_{2}-d_{12}\right)\left(x_{2}^{*}\right)^{2}\right\}$ is also satisfied. Therefore, by Theorem 18 , there is a stationary distribution (see the middle histogram in Figure 1). The left pictures in Figure 1 show that the stochastic system imitate the deterministic system. The right subgraphs are the normal quantile-quantile plots of the values of the paths $x_{1}(t)$ and $x_{2}(t)$, and they are similar to the straight lines. This means that the distribution is approximately standard normal distribution. The scatter plot of $x_{1}(t)$ and $x_{2}(t)$ is Figure 3(a); we find that almost all 


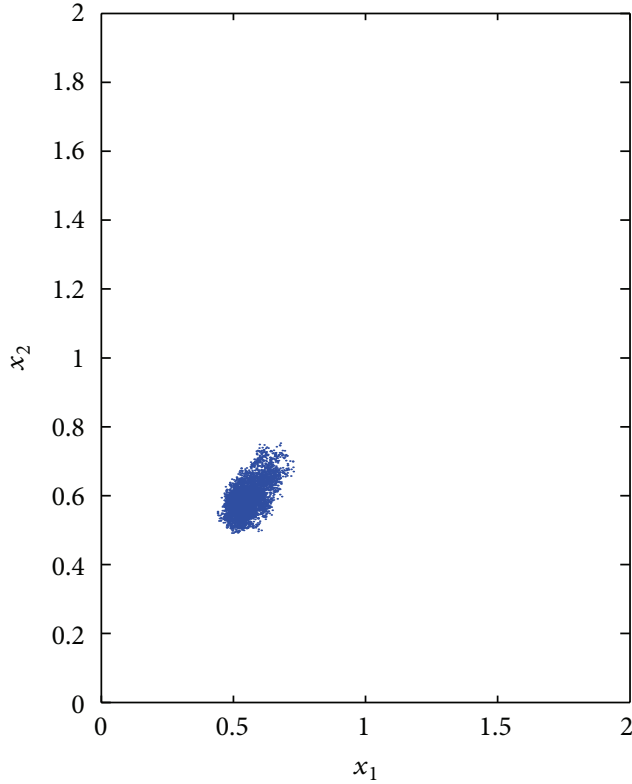

(a)

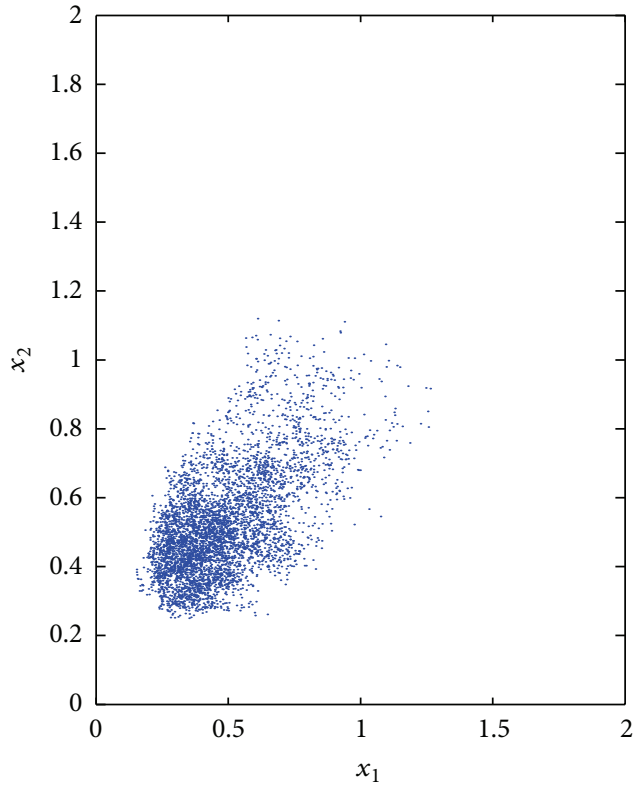

(b)

FIGURE 3: Population distribution of stochastic system (73) around the deterministic model's positive equilibrium $x^{*} \doteq(0.5734,0.6090)$. $\sigma_{1}=0.1, \sigma_{2}=0.09$ in left subgraph (a) and $\sigma_{1}=0.2, \sigma_{2}=0.3$ in the right subgraph (b).
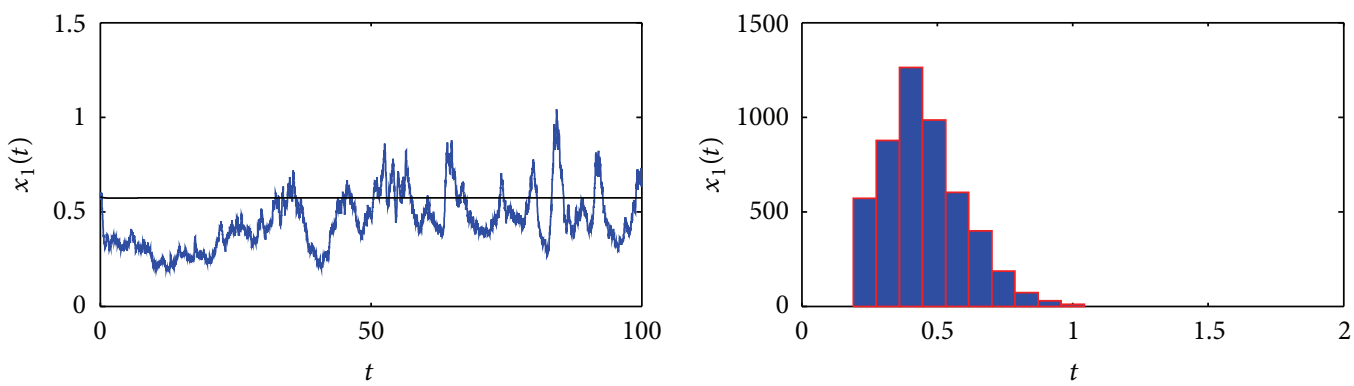

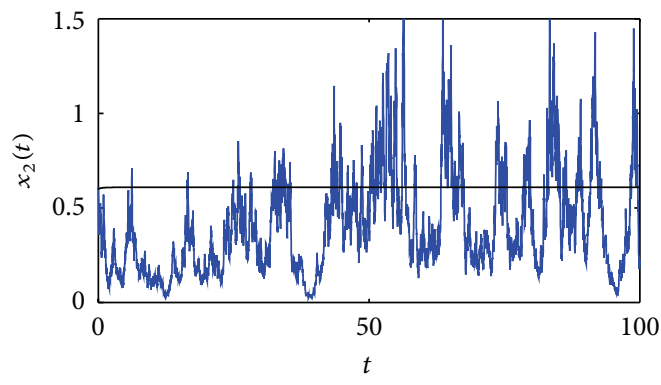

(a)

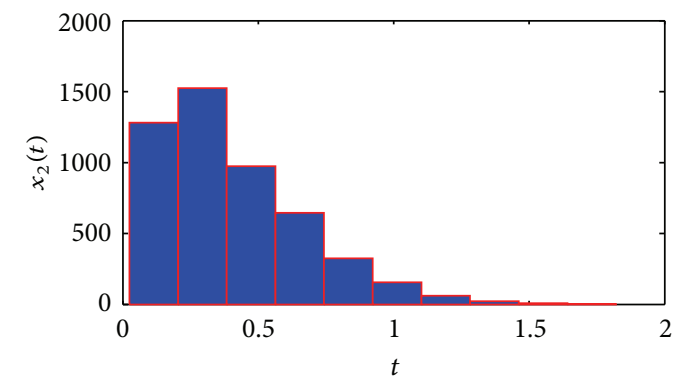

(b)

FIGURE 4: The pictures on the left are the solutions of stochastic system (73) and the corresponding undisturbed system, and the blue lines and the black lines represent them, respectively. The right subgraphs are the histogram of stochastic system $(73) . \sigma_{1}=0.2, \sigma_{2}=0.8 . d_{12}=0.6$, $d_{21}=0.5$.

population distribution lies in a small neighborhood, which can be imagined as a circular or elliptic region centered at $\left(x_{1}^{*}, x_{2}^{*}\right)$. Hence, although there is no equilibrium of the stochastic system (73) as the deterministic system, it is stochastically permanent, persistent in mean and has the ergodic property by Theorems 10, 14, and 18 .
In Figure 2, we choose $\sigma_{1}=0.2, \sigma_{2}=0.3$. The populations of $x_{1}$ and $x_{2}$ suffer relatively large white noise. By comparing Figure 1, we can see that in Figure 2 the left curves fluctuations are more violent, the histogram distribute in relatively large regions and the curves of QQ plots slightly deviate from the straight line. Comparing with Figure 3(a), 

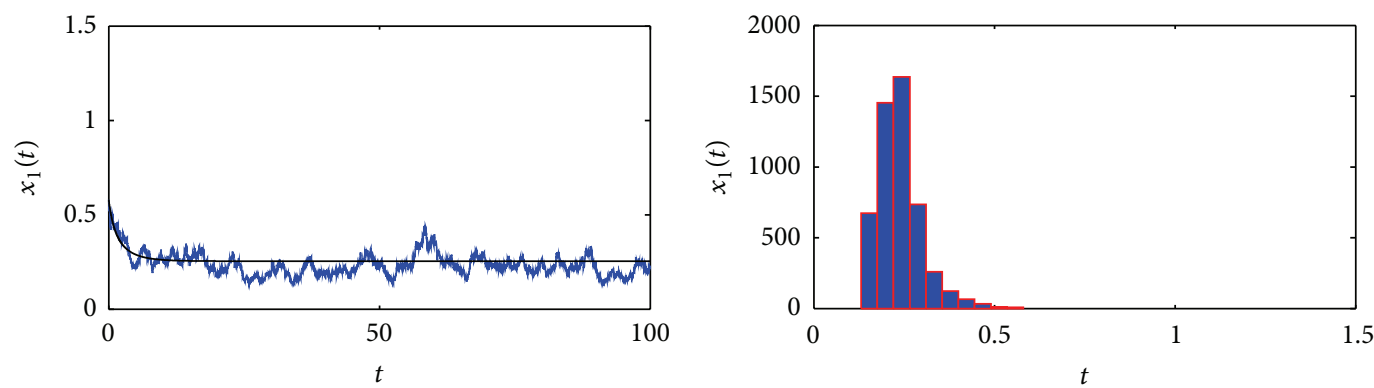

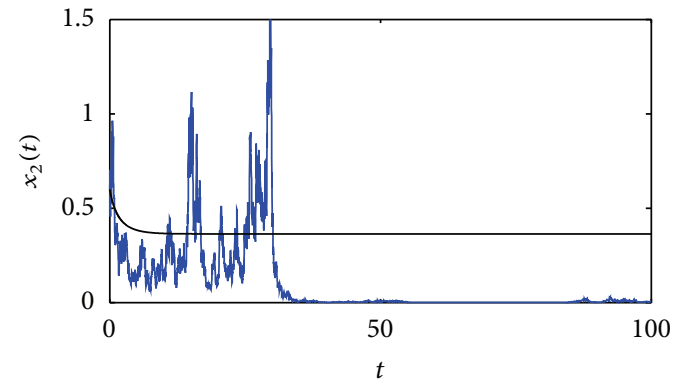

(a)

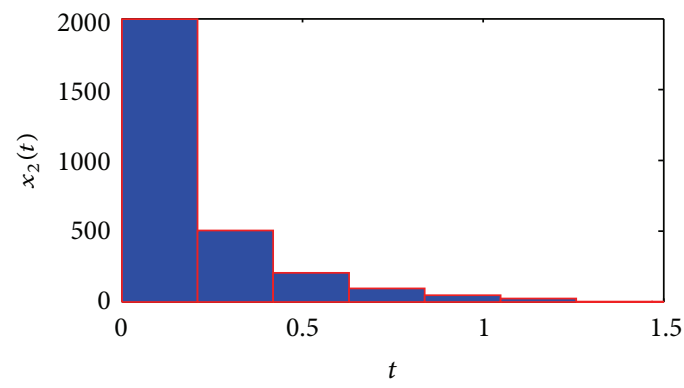

(b)

FIgURE 5: The subgraphs are defined as in Figure 4. Because there is no diffusion, $x_{2}$ is isolated and will die out; $\sigma_{1}=0.2, \sigma_{2}=0.8 ; d_{12}=0.01$, $d_{21}=0$.

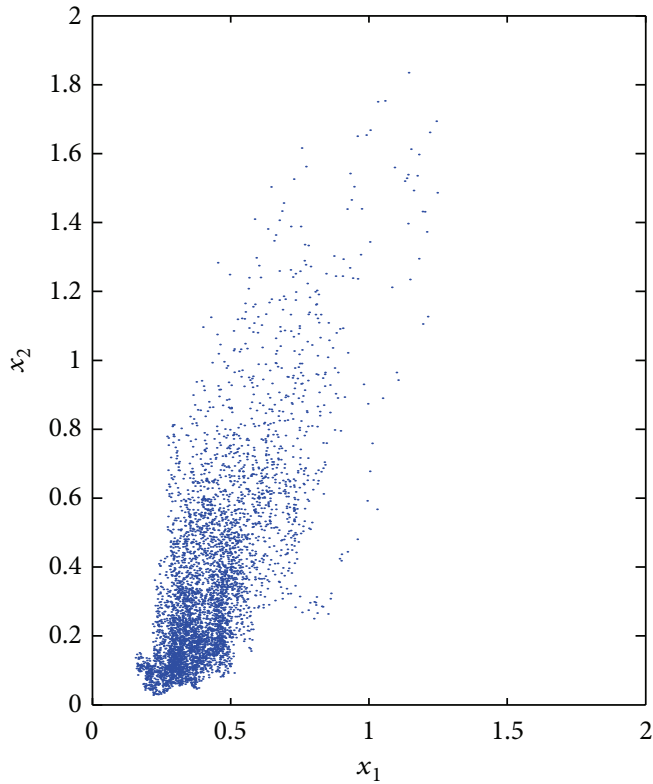

(a)

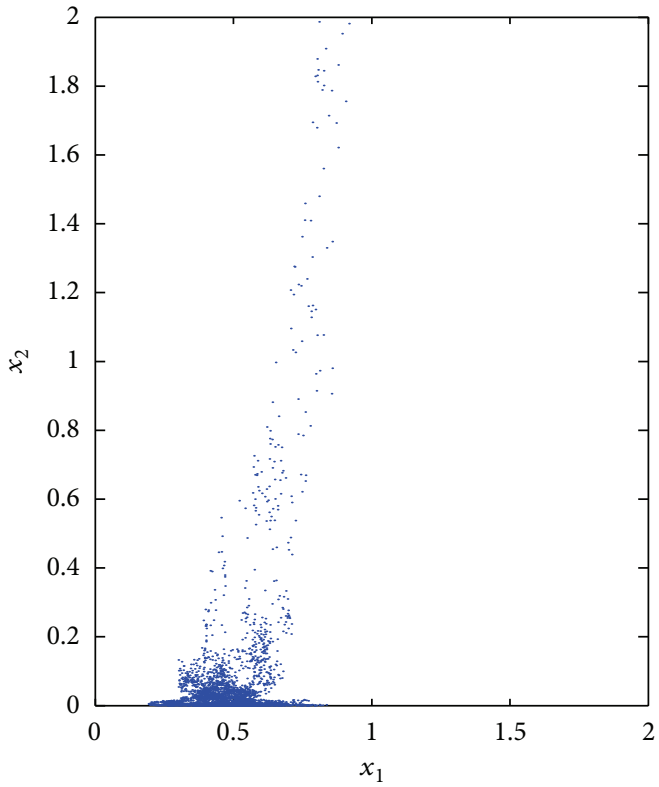

(b)

Figure 6: Population distribution of stochastic system (73); $\sigma_{1}=0.2, \sigma_{2}=0.8, d_{12}=0.6$, and $d_{21}=0.5$ in the left subgraph (a) and $\sigma_{1}=0.2$, $\sigma_{2}=0.8, d_{12}=0.01$, and $d_{21}=0$ in the right subgraph (b).

its scatter distributes in a larger area (see the scatter picture in Figure 3(b)), but we can find an ellipse to meet the condition $\delta_{2} \doteq 3.8873 \times 10^{-2}<\min \left\{\left(\bar{b}_{1}-d_{21}\right)\left(x_{1}^{*}\right)^{2},\left(\bar{b}_{2}-d_{12}\right)\left(x_{2}^{*}\right)^{2}\right\} \doteq$ 0.18544 ; by Theorems 10,14 , and 18 , we know that SDE (73) is stochastically permanent, persistent in mean and has stationary distribution.
Comparing with small white noise as in Figures 1 and 2, we choose $\sigma_{1}=0.9, \sigma_{2}=1.0$ in Figure 7. Both $x_{1}$ and $x_{2}$ suffer large white noise. We find that $a_{i}<(1 / 2) \sigma_{i}^{2}(i=1,2), \delta_{2} \doteq$ $0.5367>\min \left\{\left(\bar{b}_{1}-d_{21}\right)\left(x_{1}^{*}\right)^{2},\left(\bar{b}_{2}-d_{12}\right)\left(x_{2}^{*}\right)^{2}\right\} \doteq 0.18544$, so the conditions of Theorems 10,14, and 18 are not satisfied and the extinction conditions in Theorem 19 are satisfied, that is, 

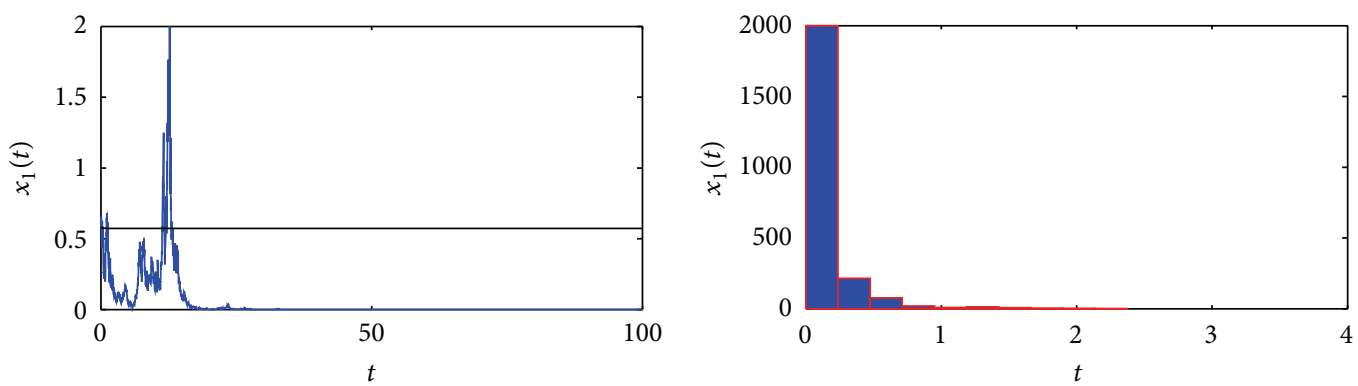

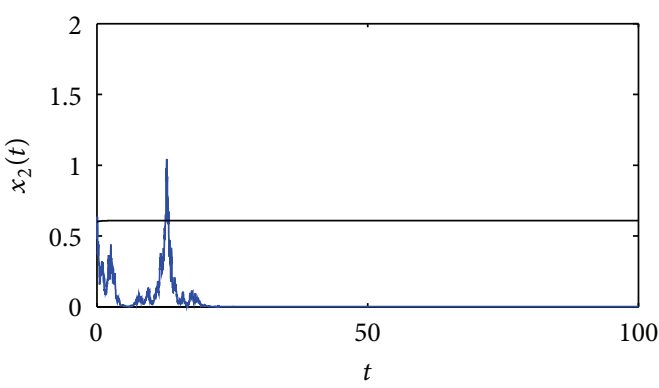

(a)

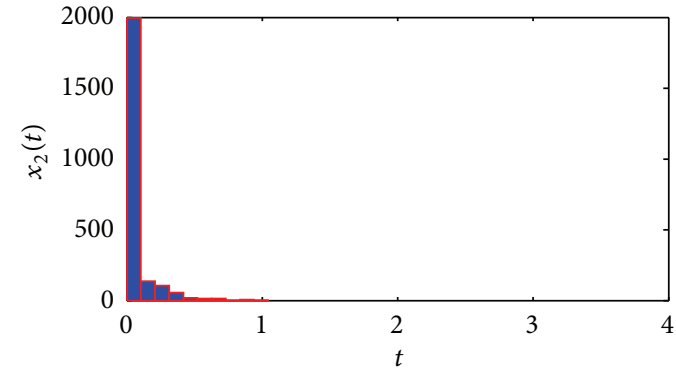

(b)

FIgURE 7: The subgraphs are defined as in Figure $4 ; \sigma_{1}=0.9, \sigma_{2}=1$. The populations of $x_{1}$ and $x_{2}$ will become extinct.

$\check{a}-\left(\widehat{\sigma}^{2} / 2\right) \doteq-4.75 \times 10^{-2}<0$; as the case in Theorem 19 expected, the species $x_{1}$ and $x_{2}$ will become extinct although the deterministic system is globally asymptotic stable.

Case 2. The effect of different diffusion coefficient on the population.

In Figure 4 , we select $\sigma_{1}=0.2, \sigma_{2}=0.8$. The conditions of Theorems 10,14 , and 18 are satisfied. $x_{2}$ suffers relatively large white noise. From the left pictures of $x_{1}(t)$ in Figures 2 and 4 , we see that the fluctuations of the two curves are different and the reason is that larger white noise of $x_{2}$ impacts $x_{1}$ in Figure 4 . In other words, due to the presence of diffusion, the relatively big white noise intensity in the individual patches will be evenly distributed to the other patches, which reduces the risk of extinction of the population. Therefore, system (73) is stochastically permanent and has a stationary distribution.

In Figure 5, we choose $d_{12}=0.01, d_{21}=0, \sigma_{1}=0.2$, and $\sigma_{2}=0.8$. Figures 4 and 5 have the same white noise intensity but have different diffusion coefficients. Because there is no diffusion effects, we can see that $x_{2}$ will die out from Figure 5 and the scatter plot Figure 6(b), that is to say, the isolated patches may become extinct if the white noise is large.

\section{Conclusion}

In this paper, we study the stochastic logistic single-species model with nonlinear directed diffusion among $n$ patches.

First, we divide the white noise intensity into small, medium, and large three cases, and through numerical simulation, we can more clearly understand the important role played by the white noise in biological populations. From these figures, we find that when the white noise is small, system (73) imitates its deterministic system and it is stochastically permanent and persistent in mean and has a stationary distribution (see Figures 1 and 3(a)). When the white noise is relatively large in some groups, it will bring relatively large deviation (see Figures 2, 3(b), 4, and 6(a)) but will not bring the species extinction due to the presence of diffusion. But, when the noise is sufficiently large in all the groups (see Figure 7), the species will become extinct even if diffusion exists. We also study the effect of different diffusion coefficient on the species and we find that isolated plaque affected by big white noise may become extinct if the diffusion coefficient is very small or equals zero (see Figures 5 and 6(b)).

In the real world, the large white noise may be bad weather, serious epidemic, which can be considered as the decisive factor responsible for the extinction of populations. Diffusion phenomena, however, play a crucial role in the development of biological populations, and human activities without control will affect the biological diffusion process which is likely to cause fatal consequences. Therefore, our research and analysis on population have great practical significance.

\section{Appendix}

In this section, we list some results about the stationary distribution (see $[15,17]$ ) which will be used in the previous sections.

Let $X(t)$ be a homogeneous Markov process in $E_{l}\left(E_{l}\right.$ denotes $l$-space) described by the stochastic equation

$$
d X(t)=b(X) d t+\sum_{r=1}^{k} g_{r}(X) d B_{r}(t)
$$


The diffusion matrix is

$$
\Lambda(x)=\left(\lambda_{i j}(x)\right), \quad \lambda_{i j}(x)=\sum_{r=1}^{k} g_{r}^{i}(x) g_{r}^{j}(x)
$$

Assumption A.1. There exists a bounded domain $U \subset E_{l}$ with regular boundary $\Gamma$, having the following properties.

(A1) In the domain $U$ and some neighborhood thereof, the smallest eigenvalue of the diffusion matrix $A(x)$ is bounded away from zero.

(B2) If $x \in E_{l} \backslash U$, the mean time $\tau$ at which a path starting from $x$ reaches the set $U$ is finite, and $\sup _{x \in K} E_{x} \tau<\infty$ for every compact subset $K \subset E_{l}$.

Lemma A.2 (see [17]). If Assumption A.1 holds, then the Markov process $X(t)$ has a stationary distribution $\mu(A)$. Let $f(\cdot)$ be a function integrable with respect to the measure $\mu$. Then

$$
P_{x}\left\{\lim _{T \rightarrow \infty} \frac{1}{T} \int_{0}^{T} f(X(t)) d t=\int_{E_{l}} f(x) \mu(d x)\right\}=1
$$

for all $x \in E_{l}$.

Remark A.3. Theorem 3 shows that there exists a unique positive solution $x(t)$ of SDE (5). Also from the proof of Theorem 3, we obtain

$$
L V \leq K
$$

Now define $\bar{V}=V+K$; then

$$
L \bar{V} \leq \bar{V}
$$

and we can get

$$
\bar{V}_{R}=\inf _{x \in R_{+}^{n} \backslash D_{m}} \bar{V}(x) \longrightarrow \infty \quad \text { as } m \longrightarrow \infty,
$$

where $D_{m}=(1 / m, m) \times(1 / m, m) \times \cdots \times(1 / m, m)$. By [17], we can obtain that the solution $x(t)$ is a homogeneous Markov process in $R_{+}^{n}$.

Lemma A.4 (see [17]). Let $x(t)$ be a regular temporally homogeneous Markov process in $E_{l}$. If $x(t)$ is recurrent relative to some bounded domain $U$, then it is recurrent relative to any nonempty domain in $E_{l}$.

\section{Conflict of Interests}

The authors declare that there is no conflict of interests regarding the publication of this paper.

\section{Acknowledgments}

The work was supported by the NSFC (no. 10971021 and no. 11001032), the Ministry of Education of China (no. 109051), the Ph.D. Programs Foundation of Ministry of China (no. 200918), and the Program for Changjiang Scholars and Innovative Research Team in University (PCSIRT).

\section{References}

[1] L. J. S. Allen, "Persistence and extinction in single-species reaction-diffusion models," Bulletin of Mathematical Biology, vol. 45, no. 2, pp. 209-227, 1983.

[2] Z. Y. Lu and Y. Takeuchi, "Global asymptotic behavior in singlespecies discrete diffusion systems," Journal of Mathematical Biology, vol. 32, no. 1, pp. 67-77, 1993.

[3] L. Allen, "Persistence, extinction, and critical patch number for island populations," Bulletin of Mathematical Biology, vol. 65, pp. 1-12, 1987.

[4] H. I. Freedman and Y. Takeuchi, "Global stability and predator dynamics in a model of prey dispersal in a patchy environment," Nonlinear Analysis: Theory, Methods \& Applications, vol. 13, no. 8, pp. 993-1002, 1989.

[5] X. R. Mao, C. G. Yuan, and J. Zou, "Stochastic differential delay equations of population dynamics," Journal of Mathematical Analysis and Applications, vol. 304, no. 1, pp. 296-320, 2005.

[6] M. Liu and K. Wang, "Persistence and extinction in stochastic non-autonomous logistic systems," Journal of Mathematical Analysis and Applications, vol. 375, no. 2, pp. 443-457, 2011.

[7] X. Y. Li, A. Gray, D. Q. Jiang, and X. R. Mao, "Sufficient and necessary conditions of stochastic permanence and extinction for stochastic logistic populations under regime switching," Journal of Mathematical Analysis and Applications, vol. 376, no. 1, pp. 11-28, 2011.

[8] D. Q. Jiang and N. Z. Shi, "A note on nonautonomous logistic equation with random perturbation," Journal of Mathematical Analysis and Applications, vol. 303, no. 1, pp. 164-172, 2005.

[9] D. Q. Jiang, N. Z. Shi, and X. Y. Li, "Global stability and stochastic permanence of a non-autonomous logistic equation with random perturbation," Journal of Mathematical Analysis and Applications, vol. 340, no. 1, pp. 588-597, 2008.

[10] C. Y. Ji, D. Q. Jiang, H. Liu, and Q. S. Yang, "Existence, uniqueness and ergodicity of positive solution of mutualism system with stochastic perturbation," Mathematical Problems in Engineering, vol. 2010, Article ID 684926, 18 pages, 2010.

[11] X. R. Mao and C. G. Yuan, Stochastic Differential Equations with Markovian Switching, Imperial College Press, London, UK, 2006.

[12] C. Ji, D. Jiang, and N. Shi, "Analysis of a predator-prey model with modified Leslie-Gower and Holling-type II schemes with stochastic perturbation," Journal of Mathematical Analysis and Applications, vol. 359, no. 2, pp. 482-498, 2009.

[13] X. R. Mao, Stochastic Differential Equations and Applications, Horwood, New York, NY, USA, 1997.

[14] N. Ikeda and S. Watanabe, Stochastic Differential Equations and Diffusion Processes, vol. 24, North-Holland, Amsterdam, The Netherlands; Kodansha Ltd., Tokyo, Japan, 2nd edition, 1989.

[15] G. Strang, Linear Algebra and Its Applications, Thomson Learning Inc., 1988.

[16] D. J. Higham, "An algorithmic introduction to numerical simulation of stochastic differential equations," SIAM Review, vol. 43, no. 3, pp. 525-546, 2001.

[17] R. Z. Hasminskii, Stochastic Stability of Differential Equations, vol. 7, Sijthoff \& Noordhoff, Alphen aan den Rijn, The Netherlands, 1980. 


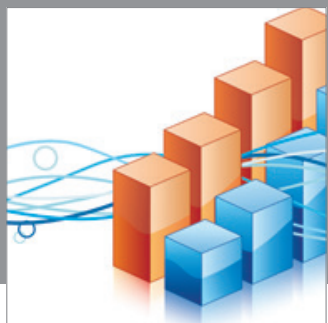

Advances in

Operations Research

mansans

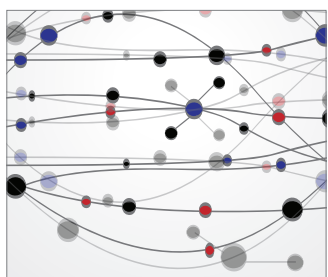

The Scientific World Journal
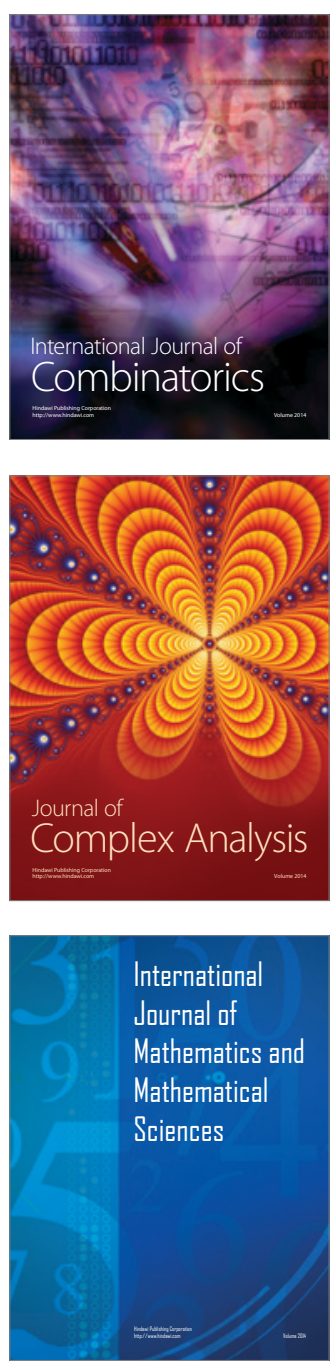
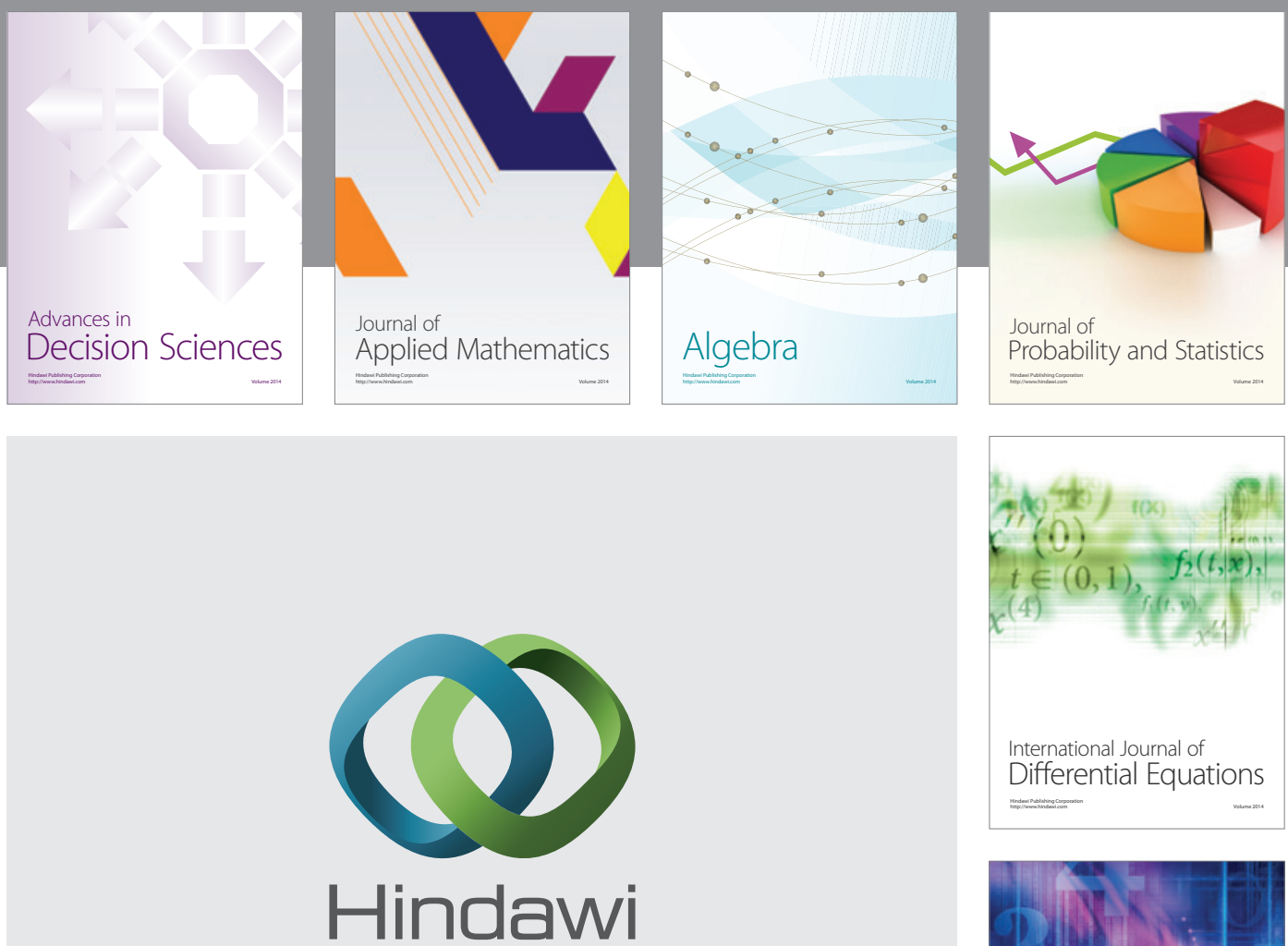

Submit your manuscripts at http://www.hindawi.com
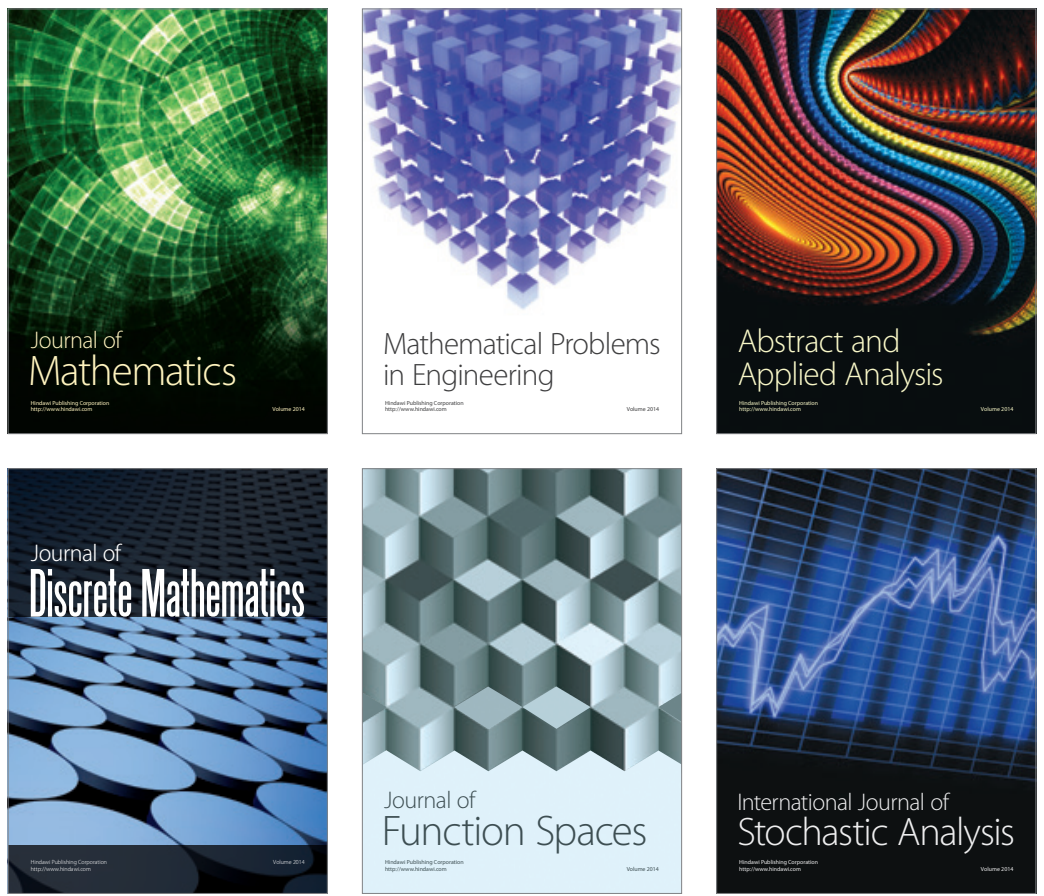

Journal of

Function Spaces

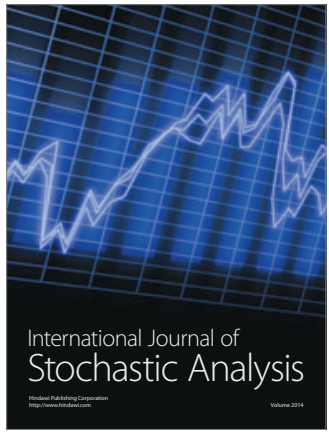

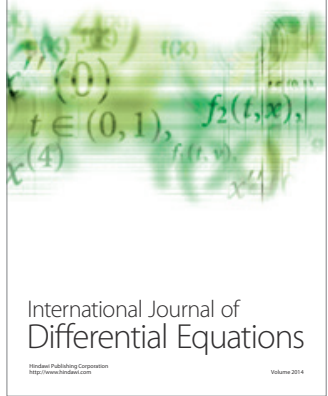
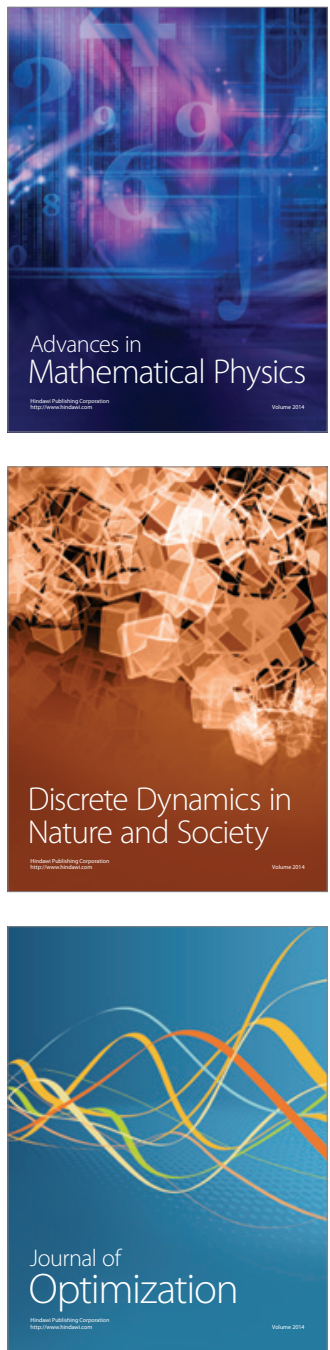